\title{
On the Pfister Number of Quadratic Forms
}

\author{
R. Parimala, V. Suresh, and J.-P. Tignol
}

\begin{abstract}
The generic quadratic form of even dimension $n$ with trivial discriminant over an arbitrary field of characteristic different from 2 containing a square root of -1 can be written in the Witt ring as a sum of 2 -fold Pfister forms using $n-2$ terms and not less. The number of 2-fold Pfister forms needed to express a quadratic form of dimension 6 with trivial discriminant is determined in various cases.
\end{abstract}

\section{Introduction}

Throughout this paper, $k$ denotes a field of characteristic different from 2 in which -1 is a square. We use the same notation for a quadratic form over $k$ and its Witt equivalence class in the Witt ring $W(k)$. As usual, the quadratic form $\sum_{i=1}^{n} a_{i} X^{i}$ with $a_{i} \in k^{\times}$is denoted by $\left\langle a_{1}, \ldots, a_{n}\right\rangle$. Since -1 is a square in $k$, the form $\langle 1, \ldots, 1\rangle$ is Witt equivalent to $\langle 1\rangle$ or 0 according as its dimension is odd or even, hence $W(k)$ is an algebra over the field $\mathbb{F}_{2}$ with two elements. Let $I(k)$ be the fundamental ideal of $W(k)$, which consists of the Witt equivalence classes of even-dimensional quadratic forms. For any integer $m \geq 1$, the $m$-th power of $I(k)$ is denoted by $I^{m}(k)$. We say a quadratic form is in $I^{m}(k)$ if its Witt equivalence class is in $I^{m}(k)$. It is well-known that for any $m \geq 1$ the ideal $I^{m}(k)$ is generated as a group by the classes of $m$-fold Pfister forms, i.e., quadratic forms of the following type:

$$
\left\langle\left\langle a_{1}, \ldots, a_{m}\right\rangle\right\rangle=\left\langle 1, a_{1}\right\rangle \otimes \cdots \otimes\left\langle 1, a_{m}\right\rangle,
$$

see [7, Prop. X.1.2]. Brosnan, Reichstein, and Vistoli [3] define the m-Pfister number $\operatorname{Pf}_{m}(q)$ of a quadratic form $q \in I^{m}(k)$ as the least number of terms in a decomposition of its Witt equivalence class into a sum of $m$-fold Pfister forms. For $m, n \geq 1$, the $(m, n)$-Pfister number $\operatorname{Pf}_{k}(m, n)$ is defined as the supremum of the $m$-Pfister numbers $\operatorname{Pf}_{m}(q)$ where $q$ runs over the quadratic forms of dimension $n$ in $I^{m}(K)$, as $K$ varies over field extensions of $k$. In [3], Pfister numbers are studied in connection with the essential dimension of algebraic groups.

A related invariant was defined by Parimala and Suresh in [9] (see also Kahn's paper [5]): the length $\lambda_{m}(q)$ of a quadratic form $q \in I^{m}(k)$ is the least integer $r$ for which there exist $m$-fold Pfister forms $\pi_{1}, \ldots, \pi_{r}$ such that $q \equiv \pi_{1}+\cdots+$ $\pi_{r} \bmod I^{m+1}(k)$. In [9], the length of quadratic forms was studied with reference

2000 Mathematics Subject Classification. 11E81.

The first author is partially supported by NSF grant DMS-0653382 and the third author is partially supported by the Fund for Scientific Research F.R.S.-FNRS (Belgium). 
to the $u$-invariant of fields and some bounds were given for the length of quadratic forms in $I^{m}(k), 1 \leq m \leq 3$. Clearly, $\lambda_{m}(q) \leq \operatorname{Pf}_{m}(q)$.

The following bounds were given in $[\mathbf{3}]$ for Pfister numbers of forms in $I(k)$ and $I^{2}(k)$ (see also Proposition 2.1 below):

Proposition ([3, Prop. 14]). $\operatorname{Pf}_{k}(1, n) \leq n$ and $\operatorname{Pf}_{k}(2, n) \leq n-2$.

Zinovy Reichstein raised the following question: Is the estimate for the 2-Pfister number in the proposition sharp, i.e., is $\operatorname{Pf}_{k}(2, n)=n-2$ ? In this paper we answer Reichstein's question in the affirmative by showing that the "generic" quadratic form $q_{0}$ of dimension $n$ with trivial discriminant satisfies $\operatorname{Pf}_{2}\left(q_{0}\right)=n-2$, see Theorem 2.2 and Corollary 2.3. Note that for any quadratic form $q$ of dimension $n$ in $I^{2}(k)$ we have $\lambda_{2}(q) \leq \frac{n-2}{2}$ (cf. [5, Prop. 1.1]); therefore for the generic form $q_{0}$ the inequality $\lambda_{2}\left(q_{0}\right) \leq \operatorname{Pf}_{2}\left(q_{0}\right)$ is strict.

The proof of Theorem 2.2 is easily derived from a discussion of a combinatorial analogue of Pfister numbers in $\S 1$. In the last section ( 33$)$, which is essentially independent from $\S \S 1$ and 2, we give some computations of Pfister numbers of quadratic forms of dimension 6 .

We are indebted to Zinovy Reichstein for his comments on a first version of this paper, which helped us to improve the wording in several points, and also to Detlev Hoffmann for suggesting an alternative proof of Theorem 2.2. Ideas from this alternative proof were used to simplify our original arguments.

\section{A combinatorial analogue}

Let $V$ be an arbitrary vector space over the field $\mathbb{F}_{2}$ with 2 elements. We consider the group algebra $\mathbb{F}_{2}[V]$ as a combinatorial analogue of the Witt ring of a field. (Indeed, the Witt ring of any field $k$ of characteristic different from 2 containing a square root of -1 is a homomorphic image of $\mathbb{F}_{2}\left[k^{\times} / k^{\times 2}\right]$, see $\S 2$.) Since the addition in $V$ is multiplication in $\mathbb{F}_{2}[V]$, it is convenient to denote by $X^{v}$ the image of $v \in V$ in $\mathbb{F}_{2}[V]$; thus

$$
\mathbb{F}_{2}[V]=\left\{\sum_{v \in V} \alpha_{v} X^{v} \mid \alpha_{v} \in \mathbb{F}_{2} \text { and }\left\{v \in V \mid \alpha_{v} \neq 0\right\} \text { is finite }\right\},
$$

with

$$
X^{0}=1 \quad \text { and } \quad X^{u} \cdot X^{v}=X^{u+v} \quad \text { for } u, v \in V .
$$

We consider the group homomorphisms

$$
\varepsilon_{0}: \mathbb{F}_{2}[V] \rightarrow \mathbb{F}_{2}, \quad \varepsilon_{1}: \mathbb{F}_{2}[V] \rightarrow V
$$

defined by

$$
\varepsilon_{0}\left(\sum_{v \in V} \alpha_{v} X^{v}\right)=\sum_{v \in V} \alpha_{v}, \quad \varepsilon_{1}\left(\sum_{v \in V} \alpha_{v} X^{v}\right)=\sum_{v \in V} \alpha_{v} v .
$$

Thus, $\varepsilon_{0}$ is the augmentation map. We denote its kernel by $I[V]$. It is an ideal since $\varepsilon_{0}$ is a ring homomorphism, and it is generated as a group by elements of the form $1+X^{v}$ for $v \in V$, which we call 1-fold Pfister elements. For $m \geq 1$, the products

$$
\left(1+X^{v_{1}}\right) \cdots\left(1+X^{v_{m}}\right) \in \mathbb{F}_{2}[V]
$$

with $v_{1}, \ldots, v_{m} \in V$ are called $m$-fold Pfister elements. They span the $m$-th power of $I[V]$, which we denote by $I^{m}[V]$ to mimic the Witt ring notation. Observe that 0 is an $m$-fold Pfister element for all $m$, since the product above is 0 if $v_{1}=0$. 
For $\xi=\sum_{v \in V} \alpha_{v} X^{v} \in \mathbb{F}_{2}[V]$ we define the support of $\xi$ by

$$
D(\xi)=\left\{v \in V \mid \alpha_{v}=1\right\} \subseteq V
$$

This notation is inspired by the usual notation for the set of represented values of a quadratic form. (See the proof of Theorem 2.2 below for an example of a field $E$ such that $W(E)$ can be identified with a group algebra $\mathbb{F}_{2}[V]$ in such a way that the support of any $\xi \in \mathbb{F}_{2}[V]$ is the set of represented values of the corresponding anisotropic quadratic form.)

LemMA 1.1. Let $\xi \in \mathbb{F}_{2}[V]$ be a nonzero element, and let $d=|D(\xi)|$ be the cardinality of the support of $\xi$.

(i) If $\xi \in I[V]$, then $d \geq 2$ and there are 1 -fold Pfister elements $\pi_{1}, \ldots, \pi_{p}$ such that

$$
\xi=\pi_{1}+\cdots+\pi_{p} \quad \text { and } \quad p \leq d
$$

If moreover $0 \in D(\xi)$, the same property holds with $p \leq d-1$.

(ii) If $\xi \in I[V]$ and $\varepsilon_{1}(\xi)=0$, then $d \geq 4$ and there exist 2-fold Pfister elements $\pi_{1}, \ldots, \pi_{p}$ such that

$$
\xi=\pi_{1}+\cdots+\pi_{p} \quad \text { and } \quad p \leq d-2 .
$$

If moreover $0 \in D(\xi)$, the same property holds with $p \leq d-3$.

Proof. (i) We have $d \neq 0$ since $\xi \neq 0$, and $d$ is even since $\varepsilon_{0}(\xi) \equiv d \bmod 2$ and $\xi \in I[V]$. Therefore, we have

$$
\xi=\sum_{v \in D(\xi)} X^{v}=\sum_{v \in D(\xi)}\left(1+X^{v}\right),
$$

proving that $\xi$ is a sum of $d$ terms that are 1-fold Pfister elements. If $0 \in D(\xi)$, one of these terms vanishes since $1+X^{0}=0$. Thus, (i) is proved.

(ii) Suppose now $\xi \in I[V]$ and $\varepsilon_{1}(\xi)=0$. As in case (i), $d$ is even. If $d=2$, the condition $\varepsilon_{1}(\xi)=0$ yields $\xi=0$. Therefore, $d \geq 4$. The other assertions are proved by induction on $d$. Suppose first $0 \in D(\xi)$. Since $d \geq 4$ we may find in $D(\xi)$ two distinct nonzero vectors $u, v$. Define

$$
\xi^{\prime}=\left(1+X^{u}\right)\left(1+X^{v}\right)+\xi
$$

We have $\xi^{\prime} \in I[V]$ and $\varepsilon_{1}\left(\xi^{\prime}\right)=0$. Moreover,

$$
D\left(\xi^{\prime}\right) \subseteq(D(\xi) \backslash\{0, u, v\}) \cup\{u+v\},
$$

hence $\left|D\left(\xi^{\prime}\right)\right| \leq d-2$. By induction, there exist 2-fold Pfister elements $\pi_{1}, \ldots, \pi_{p}$ such that

$$
\xi^{\prime}=\pi_{1}+\cdots+\pi_{p} \quad \text { and } \quad p \leq d-4,
$$

$\left(\xi^{\prime}=0\right.$ if $\left.d=4\right)$. Then

$$
\xi=\pi_{1}+\cdots+\pi_{p}+\left(1+X^{u}\right)\left(1+X^{v}\right)
$$

and the number of terms on the right side is at most $d-3$. If $0 \notin D(\xi)$ we may still define $\xi^{\prime}$ as above, and we have

$$
0 \in D\left(\xi^{\prime}\right) \subseteq(D(\xi) \backslash\{u, v\}) \cup\{0, u+v\},
$$

hence $\left|D\left(\xi^{\prime}\right)\right| \leq d$. The arguments above show that there exist 2-fold Pfister elements $\pi_{1}, \ldots, \pi_{p}$ such that

$$
\xi^{\prime}=\pi_{1}+\cdots+\pi_{p} \quad \text { and } \quad p \leq d-3 .
$$


Then

$$
\xi=\pi_{1}+\cdots+\pi_{p}+\left(1+X^{u}\right)\left(1+X^{v}\right)
$$

and the number of terms on the right side is at most $d-2$.

Corollary 1.2. $I^{2}[V]=\left\{\xi \in I[V] \mid \varepsilon_{1}(\xi)=0\right\}$.

Proof. Lemma 1.1(i) shows that $I[V]$ is spanned by 1-fold Pfister elements, hence $I^{2}[V]$ is generated as a group by 2-fold Pfister elements. Since these elements lie in the kernel of $\varepsilon_{1}$, it follows that

$$
I^{2}[V] \subseteq\left\{\xi \in I[V] \mid \varepsilon_{1}(\xi)=0\right\} .
$$

The reverse inclusion readily follows from Lemma 1.1(ii).

For $\xi \in I^{m}[V]$ we define the $m$-Pfister number $\operatorname{Pf}_{m}(\xi)$ as the minimal number of terms in a decomposition of $\xi$ as a sum of $m$-fold Pfister elements. In particular, $\mathrm{Pf}_{m}(0)=0$ for all $m \geq 1$.

Proposition 1.3. For every $\xi \in I[V]$ we have

$$
\operatorname{Pf}_{1}(\xi)= \begin{cases}|D(\xi)| & \text { if } 0 \notin D(\xi), \\ |D(\xi)|-1 & \text { if } 0 \in D(\xi)\end{cases}
$$

Proof. Let $p=\operatorname{Pf}_{1}(\xi)$. Suppose $v_{1}, \ldots, v_{p} \in V$ are nonzero vectors such that

$$
\xi=\sum_{i=1}^{p}\left(1+X^{v_{i}}\right)=p+\sum_{i=1}^{p} X^{v_{i}} .
$$

Then $D(\xi) \subseteq\left\{0, v_{1}, \ldots, v_{p}\right\}$, hence

$$
|D(\xi)| \leq \begin{cases}p & \text { if } 0 \notin D(\xi), \\ p+1 & \text { if } 0 \in D(\xi) .\end{cases}
$$

The reverse inequality follows from Lemma 1.1.

We now turn to 2-Pfister numbers. From Lemma 1.1 it follows that for $\xi \neq 0$ in $I^{2}[V]$,

$$
\operatorname{Pf}_{2}(\xi) \leq|D(\xi)|-2, \quad \text { and } \quad \operatorname{Pf}_{2}(\xi) \leq|D(\xi)|-3 \text { if } 0 \in D(\xi)
$$

In the rest of this section, we explicitly construct elements for which the upper bound is reached. The following general observation is crucial for the proof: every linear map $\varphi: V \rightarrow W$ between $\mathbb{F}_{2}$-vector spaces induces a ring homomorphism $\varphi_{*}: \mathbb{F}_{2}[V] \rightarrow \mathbb{F}_{2}[W]$ by

$$
\varphi_{*}\left(\sum_{v \in V} \alpha_{v} X^{v}\right)=\sum_{v \in V} \alpha_{v} X^{\varphi(v)} .
$$

The homomorphism $\varphi_{*}$ maps 1-fold Pfister elements in $\mathbb{F}_{2}[V]$ to (possibly zero) 1-fold Pfister elements in $\mathbb{F}_{2}[W]$, hence also $m$-fold Pfister elements in $\mathbb{F}_{2}[V]$ to $m$ fold Pfister elements in $\mathbb{F}_{2}[W]$, for every $m \geq 1$. Consequently, for every $\xi \in I^{m}[V]$ we have $\varphi_{*}(\xi) \in I^{m}[W]$ and

$$
\operatorname{Pf}_{m}\left(\varphi_{*}(\xi)\right) \leq \operatorname{Pf}_{m}(\xi)
$$


Now, let $V$ be an $\mathbb{F}_{2}$-vector space of finite dimension $n>1$, and let $e=\left(e_{i}\right)_{i=1}^{n}$ be a base of $V$. We define $e_{0}=\sum_{i=1}^{n} e_{i}$ and

$$
\xi_{e}=n+1+\sum_{i=0}^{n} X^{e_{i}} \in \mathbb{F}_{2}[V] .
$$

It is readily verified that $\varepsilon_{0}(\xi)=0$ and $\varepsilon_{1}(\xi)=0$, so $\xi_{e} \in I^{2}[V]$, and the support of $\xi_{e}$ is

$$
D\left(\xi_{e}\right)= \begin{cases}\left\{0, e_{0}, e_{1}, \ldots, e_{n}\right\} & \text { if } n \text { is even, } \\ \left\{e_{0}, e_{1}, \ldots, e_{n}\right\} & \text { if } n \text { is odd. }\end{cases}
$$

Therefore, (1) yields the same inequality when $n$ is odd or even:

$$
\mathrm{Pf}_{2}\left(\xi_{e}\right) \leq n-1 \text {. }
$$

The following proposition shows that $\mathrm{Pf}_{2}\left(\xi_{e}\right)$ reaches the bound in (1).

Proposition 1.4. $\operatorname{Pf}_{2}\left(\xi_{e}\right)=n-1$.

Proof. We use induction on $n$. If $n=2$, we have

$$
\xi_{e}=\left(1+X^{e_{1}}\right)\left(1+X^{e_{2}}\right)
$$

so $\operatorname{Pf}_{2}\left(\xi_{e}\right)=1$. If $n=3$, then

$$
\xi_{e}=X^{e_{1}}+X^{e_{2}}+X^{e_{3}}+X^{e_{1}} X^{e_{2}} X^{e_{3}} \text {. }
$$

This element is not a 2 -fold Pfister element since $0 \notin D\left(\xi_{e}\right)$, hence $\operatorname{Pf}_{2}\left(\xi_{e}\right)>1$. On the other hand, $\operatorname{Pf}_{2}\left(\xi_{e}\right) \leq 2$ by $(3)$, hence $\operatorname{Pf}_{2}\left(\xi_{e}\right)=2$.

For the rest of the proof, suppose $n>3$. Let $p=\operatorname{Pf}_{2}\left(\xi_{e}\right)$ and let $\pi_{1}, \ldots, \pi_{p}$ be 2-fold Pfister elements such that

$$
\xi_{e}=\pi_{1}+\cdots+\pi_{p}
$$

We have $D\left(\xi_{e}\right) \subseteq \bigcup_{i=1}^{p} D\left(\pi_{i}\right)$, hence $e_{n} \in D\left(\pi_{i}\right)$ for some $i=1, \ldots, p$. Renumbering, we may assume $e_{n} \in D\left(\pi_{p}\right)$, hence

$$
\pi_{p}=1+X^{e_{n}}+X^{v}+X^{e_{n}+v} \quad \text { for some } v \in V .
$$

Let $W \subseteq V$ be the $\mathbb{F}_{2}$-span of $e_{1}, \ldots, e_{n-1}$, and let $f_{0}=\sum_{i=1}^{n-1} e_{i} \in W$. Clearly, $f=\left(e_{i}\right)_{i=1}^{n-1}$ is a base of $W$, and the element $\xi_{f} \in \mathbb{F}_{2}[W]$ built on the same model as $\xi_{e}$ is

$$
\xi_{f}=n+X^{f_{0}}+\sum_{i=1}^{n-1} X^{e_{i}}
$$

Consider the linear map $\varphi: V \rightarrow W$ defined by

$$
\varphi\left(e_{i}\right)= \begin{cases}e_{i} & \text { for } i=1, \ldots, n-1, \\ 0 & \text { for } i=n .\end{cases}
$$

The ring homomorphism $\varphi_{*}: \mathbb{F}_{2}[V] \rightarrow \mathbb{F}_{2}[W]$ induced by $\varphi$ as in (2) above satisfies $\varphi_{*}\left(X^{e_{n}}\right)=1$. Since $\varphi\left(e_{0}\right)=f_{0}$, it follows that $\varphi_{*}\left(\xi_{e}\right)=\xi_{f}$, hence (4) yields

$$
\xi_{f}=\varphi_{*}\left(\pi_{1}\right)+\cdots+\varphi_{*}\left(\pi_{p}\right)
$$

In view of $(5)$, we have $\varphi_{*}\left(\pi_{p}\right)=0$, hence the preceding equation shows that $\operatorname{Pf}_{2}\left(\xi_{f}\right) \leq p-1$. Since $\operatorname{dim} W=n-1$, the induction hypothesis yields $\operatorname{Pf}_{2}\left(\xi_{f}\right)=$ $n-2$, hence $n-1 \leq p$. The reverse inequality holds by (3), hence the proposition is proved. 


\section{Pfister numbers of generic forms}

Let $k$ be an arbitrary field of characteristic different from 2 containing a square root of -1 , and let $V_{k}=k^{\times} / k^{\times 2}$ be the group of square classes in $k$, which we view as an $\mathbb{F}_{2}$-vector space. The map

$$
\Psi: V_{k} \rightarrow W(k)
$$

defined by $\Psi\left(a k^{\times 2}\right)=\langle a\rangle$ for $a \in k^{\times}$is multiplicative, hence it induces a surjective $\mathbb{F}_{2}$-algebra homomorphism

$$
\Psi_{*}: \mathbb{F}_{2}\left[V_{k}\right] \rightarrow W(k)
$$

The map $\Psi_{*}$ carries 1-fold Pfister elements in $\mathbb{F}_{2}\left[V_{k}\right]$ to 1-fold Pfister forms in $W(k)$, hence also $m$-fold Pfister elements to $m$-fold Pfister forms for all $m \geq 1$. Therefore, $\Psi_{*}\left(I^{m}\left[V_{k}\right]\right)=I^{m}(k)$ and we have

$$
\operatorname{Pf}_{m}\left(\Psi_{*}(\xi)\right) \leq \operatorname{Pf}_{m}(\xi) \quad \text { for all } \xi \in I^{m}\left[V_{k}\right] .
$$

We may then use Lemma 1.1 to give a short proof of Proposition 14 of [3], including a minor refinement:

Proposition 2.1. Let $q$ be a quadratic form of dimension $n$ over a field $k$ containing a square root of -1 .

(i) If $q \in I(k)$, then $\operatorname{Pf}_{1}(q) \leq n$. If moreover $q$ represents 1 , then $\operatorname{Pf}_{1}(q) \leq$ $n-1$.

(ii) If $q \in I^{2}(k)$, then $\operatorname{Pf}_{2}(q) \leq n-2$. If moreover $q$ represents 1 , then $\mathrm{Pf}_{2}(q) \leq n-3$.

Proof. Let $q=\left\langle a_{1}, \ldots, a_{n}\right\rangle$. Consider then

$$
\xi=\left(a_{1} k^{\times 2}\right)+\cdots+\left(a_{n} k^{\times 2}\right) \in \mathbb{F}_{2}\left[V_{k}\right] .
$$

We have $\Psi_{*}(\xi)=q$ and $D(\xi)=\left\{a_{1} k^{\times 2}, \ldots, a_{n} k^{\times 2}\right\}$, so $|D(\xi)| \leq n$. If $q \in I(k)$, then $n$ is even hence $\xi \in I\left[V_{k}\right]$. Lemma 1.1(i) then yields $\operatorname{Pf}_{1}(\xi) \leq n$, and by (6) it follows that $\mathrm{Pf}_{1}(q) \leq n$. If $q$ represents 1 , then we may assume $a_{1}=1$, hence $D(\xi)$ contains the zero element of $V_{k}$. Lemma 1.1(i) then yields $\operatorname{Pf}_{1}(\xi) \leq n-1$, and by (6) it follows that $\operatorname{Pf}_{1}(q) \leq n-1$.

If $q \in I^{2}(k)$, then $a_{1} \ldots a_{n} \in k^{\times 2}$ hence $\varepsilon_{1}(\xi)=0$. By Corollary 1.2 we have $\xi \in I^{2}\left[V_{k}\right]$, and Lemma 1.1(ii) yields $\operatorname{Pf}_{2}(\xi) \leq n-2$. Therefore, by (6) we get $\operatorname{Pf}_{2}(q) \leq n-2$. Again, if $q$ represents 1 we may assume $0 \in D(\xi)$, and the preceding inequalities can be strengthened to

$$
\mathrm{Pf}_{2}(q) \leq \mathrm{Pf}_{2}(\xi) \leq n-3 .
$$

For the rest of this section, fix an arbitrary integer $n \geq 2$. Consider $n$ independent indeterminates $x_{1}, \ldots, x_{n}$ over $k$ and let

$$
x_{0}=x_{1} \cdots x_{n} .
$$

Over the field $K=k\left(x_{1}, \ldots, x_{n}\right)$, we consider the following quadratic forms:

$$
\begin{aligned}
q & =\left\langle x_{1}, \ldots, x_{n}\right\rangle, & & q_{0}=\left\langle x_{0}, x_{1}, \ldots, x_{n}\right\rangle, \\
q^{\prime} & =\left\langle 1, x_{1}, \ldots, x_{n}\right\rangle, & & q_{0}^{\prime}=\left\langle 1, x_{0}, x_{1}, \ldots, x_{n}\right\rangle .
\end{aligned}
$$

If $n$ is even, then $q \in I(K)$ and $q_{0}^{\prime} \in I^{2}(K)$. If $n$ is odd, then $q^{\prime} \in I(K)$ and $q_{0} \in I^{2}(K)$. 
THEOREM 2.2. If $n$ is even, then

$$
\operatorname{Pf}_{1}(q)=n \quad \text { and } \quad \operatorname{Pf}_{2}\left(q_{0}^{\prime}\right)=n-1 .
$$

If $n$ is odd, then

$$
\operatorname{Pf}_{1}\left(q^{\prime}\right)=n \quad \text { and } \quad \operatorname{Pf}_{2}\left(q_{0}\right)=n-1 .
$$

PROOF. Let $k_{\text {alg }}$ be an algebraic closure of $k$. Embed $k$ in the field of iterated Laurent series $E=k_{\text {alg }}\left(\left(x_{1}\right)\right) \cdots\left(\left(x_{n}\right)\right)$. Applying Springer's theorem in [7, Cor. VI.1.7] recursively, we obtain a ring isomorphism

$$
\Theta: W(E) \stackrel{\sim}{\rightarrow} \mathbb{F}_{2}\left[(\mathbb{Z} / 2 \mathbb{Z})^{n}\right],
$$

which maps $W\left(k_{\text {alg }}\right)$ onto $\mathbb{F}_{2}$ and maps the quadratic form $\left\langle x_{i}\right\rangle$ to $X^{e_{i}}$, where $e_{i}$ is the $i$-th element in the standard base of $(\mathbb{Z} / 2 \mathbb{Z})^{n}$ as an $\mathbb{F}_{2}$-vector space, for $i=1$, $\ldots, n$. Note that the $\left(x_{1}, \ldots, x_{n}\right)$-adic valuation on $E$ yields an isomorphism

$$
V_{E}=E^{\times} / E^{\times 2} \simeq(\mathbb{Z} / 2 \mathbb{Z})^{n}
$$

which maps $x_{i} E^{\times 2}$ to $e_{i}$ for $i=1, \ldots, n$. Using this isomorphism as an identification, we may view $\Theta$ as the inverse map of $\Psi_{*}: \mathbb{F}_{2}\left[V_{E}\right] \rightarrow W(E)$, which is thus an isomorphism in this case.

Letting $e_{0}=\sum_{i=1}^{n} e_{i}$, we have

$$
\begin{array}{ll}
\Theta\left(q_{E}\right)=\sum_{i=1}^{n} X^{e_{i}}, & \Theta\left(q_{0 E}\right)=\sum_{i=0}^{n} X^{e_{i}}, \\
\Theta\left(q_{E}^{\prime}\right)=1+\sum_{i=1}^{n} X^{e_{i}}, & \Theta\left(q_{0 E}^{\prime}\right)=1+\sum_{i=0}^{n} X^{e_{i}},
\end{array}
$$

hence in the notation of $\S 1$ with $V=(\mathbb{Z} / 2 \mathbb{Z})^{n}$, we have

$$
\xi_{e}= \begin{cases}\Theta\left(q_{0 E}\right) & \text { if } n \text { is odd } \\ \Theta\left(q_{0 E}^{\prime}\right) & \text { if } n \text { is even. }\end{cases}
$$

The isomorphism $\Theta$ maps $m$-fold Pfister forms in $W(E)$ to $m$-fold Pfister elements in $\mathbb{F}_{2}[V]$, hence it preserves $m$-Pfister numbers. Therefore, Proposition 1.4 yields

$$
\operatorname{Pf}_{2}\left(q_{0 E}\right)=n-1 \quad \text { if } n \text { is odd } \quad \text { and } \quad \operatorname{Pf}_{2}\left(q_{0 E}^{\prime}\right)=n-1 \text { if } n \text { is even. }
$$

Similarly, Proposition 1.3 yields

$$
\operatorname{Pf}_{1}\left(q_{E}\right)=n \quad \text { if } n \text { is even } \quad \text { and } \quad \operatorname{Pf}_{1}\left(q_{E}^{\prime}\right)=n \quad \text { if } n \text { is odd. }
$$

Since scalar extension preserves $m$-Pfister forms, it follows that

$$
\begin{aligned}
& \operatorname{Pf}_{1}(q) \geq n \quad \text { and } \quad \operatorname{Pf}_{2}\left(q_{0}^{\prime}\right) \geq n-1 \quad \text { if } n \text { is even, } \\
& \operatorname{Pf}_{1}\left(q^{\prime}\right) \geq n \quad \text { and } \quad \operatorname{Pf}_{2}\left(q_{0}\right) \geq n-1 \quad \text { if } n \text { is odd. }
\end{aligned}
$$

The reverse inequalities follow from Proposition 2.1.

Corollary 2.3. $\operatorname{Pf}_{k}(1, m)=m$ for any even integer $m \geq 2$ and $\operatorname{Pf}_{k}(2, m)=$ $m-2$ for any even integer $m \geq 4$.

Proof. For $m$ even, $m \geq 2$, the form $q$ above (with $n=m$ ) has dimension $m$ and satisfies $q \in I(F)$ and $\operatorname{Pf}_{1}(q)=m$, so $\operatorname{Pf}_{k}(1, m) \geq m$. Similarly, for $m$ even, $m \geq 4$, the form $q_{0}$ above (with $n=m-1$ ) has dimension $m$ and satisfies $q_{0} \in I^{2}(\bar{F})$ and $\operatorname{Pf}_{2}\left(q_{0}\right)=m-2$, so $\operatorname{Pf}_{k}(2, m) \geq m-2$. The reverse inequalities follow from [3, Prop. 14] (see the Introduction or Proposition 2.1). 
R. PARIMALA, V. SURESH, AND J.-P. TIGNOL

REMARK 2.4. A form with the same 2-Pfister number as $q_{0}^{\prime}$ can be obtained by scaling $q_{0}$ : we have

$$
\left\langle x_{1}\right\rangle q_{0}=\left\langle 1, x_{1} x_{2}, \ldots, x_{1} x_{n}, x_{0} x_{1}\right\rangle
$$

and $x_{1} x_{2}, \ldots, x_{1} x_{n}$ may be regarded as independent indeterminates. If $n$ is odd we have

$$
x_{0} x_{1} \equiv\left(x_{1} x_{2}\right) \cdots\left(x_{1} x_{n}\right) \bmod K^{\times 2},
$$

hence $\left\langle x_{1}\right\rangle q_{0}$ is isometric to a quadratic form like $q_{0}^{\prime}$ in the indeterminates $x_{1} x_{2}, \ldots$, $x_{1} x_{n}$. Embedding $K$ in $E$ as in the proof of Theorem 2.2, we obtain $\operatorname{Pf}_{2}\left(\left\langle x_{1}\right\rangle q_{0}\right)=$ $n-2$. Details are left to the reader.

\section{Low-dimensional forms}

Let $k$ be an arbitrary field of characteristic different from 2 containing a square root of -1 . In this section, we obtain some information on the 2-Pfister number of quadratic forms of dimension 4 or 6 over $k$.

The case of anisotropic quadratic forms $q \in I^{2}(k)$ of dimension 4 is clear: if $q$ represents 1 , then $q$ is a 2 -fold Pfister form, so $\operatorname{Pf}_{2}(q)=1$. On the other hand, if $q$ does not represent 1, then $\operatorname{Pf}_{2}(q)>1$ and Proposition 2.1 yields $\operatorname{Pf}_{2}(q)=2$.

We next consider anisotropic forms of dimension 6 in $I^{2}(k)$. Of course, $\mathrm{Pf}_{2}(q)>$ 1 for any such form $q$. If $q$ represents 1, it follows from Proposition 2.1 that $\mathrm{Pf}_{2}(q)=2$ or 3 . The Stiefel-Whitney invariant $w_{4}(q) \in H^{4}\left(k, \mu_{2}\right)$ discriminates between the two cases, as the next proposition shows. (See $[\mathbf{8}, \S 3]$ or $[\mathbf{4}, \S 17]$ for a discussion of Stiefel-Whitney invariants of quadratic forms.)

Proposition 3.1. Let $q$ be an anisotropic quadratic form of dimension 6 . Assume $q \in I^{2}(k)$ and $q$ represents 1 . If $w_{4}(q)=0$, then $\operatorname{Pf}_{2}(q)=2$. If $w_{4}(q) \neq 0$, then $\operatorname{Pf}_{2}(q)=3$.

ProOF. In view of Proposition 2.1, it suffices to show that $\operatorname{Pf}_{2}(q)=2$ if and only if $w_{4}(q)=0$. Assume first $\operatorname{Pf}_{2}(q)=2$ so that

$$
q=\left\langle x_{1}, x_{2}, x_{1} x_{2}, y_{1}, y_{2}, y_{1} y_{2}\right\rangle \quad \text { for some } x_{1}, \ldots, y_{4} \in k^{\times} .
$$

For $x \in k^{\times}$, denote by $(x) \in H^{1}\left(k, \mu_{2}\right)$ the cohomology class associated to the square class of $x$. An explicit computation yields

$$
w_{4}(q)=\left(x_{1}\right) \cup\left(x_{2}\right) \cup\left(y_{1}\right) \cup\left(y_{2}\right) .
$$

Since $q$ represents 1 , the form $\langle 1\rangle \perp q$ is isotropic. The 4 -fold Pfister form $\left\langle\left\langle x_{1}, x_{2}, y_{1}, y_{2}\right\rangle\right\rangle$ which contains $\langle 1\rangle \perp q$ as a subform is hyperbolic. Therefore, $\left(x_{1}\right) \cup\left(x_{2}\right) \cup\left(y_{1}\right) \cup\left(y_{2}\right)=0$ by $[\mathbf{1}$, Satz 1.6].

For the converse, let

$$
q=\langle 1, a, b, c, d, a b c d\rangle \quad \text { for some } a, b, c, d \in k^{\times} .
$$

Then

$$
w_{4}(q)=(a) \cup(b) \cup(c) \cup(d) .
$$

Since $w_{4}(q)=0$ by hypothesis, Theorem 1 of $[\mathbf{2}]$ shows that the 4 -fold Pfister form $\langle\langle a, b, c, d\rangle\rangle$ is hyperbolic. It follows that the 9-dimensional subform $q \perp\langle a b, a c, a d\rangle$ is isotropic, hence $q$ represents a nonzero element of the form $a\left(b x^{2}+c y^{2}+d z^{2}\right)$ for 
some $x, y, z \in k$. Let $b^{\prime}=b x^{2}+c y^{2}+d z^{2} \in k^{\times}$. Since the form $\langle b, c, d\rangle$ represents $b^{\prime}$, we may find $c^{\prime}, d^{\prime} \in k^{\times}$such that

$$
\langle b, c, d\rangle=\left\langle b^{\prime}, c^{\prime}, d^{\prime}\right\rangle .
$$

Comparing discriminants, we have $b c d \equiv b^{\prime} c^{\prime} d^{\prime} \bmod k^{\times 2}$, hence

$$
q=\left\langle 1, a, b^{\prime}, c^{\prime}, d^{\prime}, a b^{\prime} c^{\prime} d^{\prime}\right\rangle \text {. }
$$

The form $\left\langle 1, a, b^{\prime}\right\rangle$ is anisotropic since $q$ is anisotropic, hence the 2-fold Pfister form $\left\langle\left\langle a, b^{\prime}\right\rangle\right\rangle$ is anisotropic. On the other hand, the form $q \perp\left\langle a b^{\prime}\right\rangle$ is isotropic since $q$ represents $a b^{\prime}$, hence $\left\langle\left\langle a, b^{\prime}\right\rangle\right\rangle$ represents a nonzero element of the form $c^{\prime} r^{2}+d^{\prime} s^{2}+$ $a b^{\prime} c^{\prime} d^{\prime} t^{2}$, for some $r, s, t \in k$. Let $c^{\prime \prime}=c^{\prime} r^{2}+d^{\prime} s^{2}+a b^{\prime} c^{\prime} d^{\prime} t^{2} \in k^{\times}$, and let $d^{\prime \prime} \in k^{\times}$ be such that

$$
\left\langle c^{\prime}, d^{\prime}, a b^{\prime} c^{\prime} d^{\prime}\right\rangle=\left\langle c^{\prime \prime}, d^{\prime \prime}, a b^{\prime} c^{\prime \prime} d^{\prime \prime}\right\rangle
$$

Thus

$$
q=\left\langle 1, a, b^{\prime}, c^{\prime \prime}, d^{\prime \prime}, a b^{\prime} c^{\prime \prime} d^{\prime \prime}\right\rangle .
$$

Since $\left\langle\left\langle a, b^{\prime}\right\rangle\right\rangle$ represents $c^{\prime \prime}$, the 3 -fold Pfister form $\left\langle\left\langle a, b^{\prime}, c^{\prime \prime}\right\rangle\right\rangle$ is hyperbolic, and therefore its 5-dimensional subform $\left\langle 1, a, b^{\prime}, c^{\prime \prime}, a b^{\prime} c^{\prime \prime}\right\rangle$ is isotropic. Thus, $\left\langle 1, a, b^{\prime}, c^{\prime \prime}\right\rangle$ represents $a b^{\prime} c^{\prime \prime}$, and we may find $u, v \in k^{\times}$such that

$$
\left\langle 1, a, b^{\prime}, c^{\prime \prime}\right\rangle=\left\langle a b^{\prime} c^{\prime \prime}, u, v, u v\right\rangle .
$$

Thus

and $\operatorname{Pf}_{2}(q)=2$.

$$
q=\langle\langle u, v\rangle\rangle+\left\langle\left\langle d^{\prime \prime}, a b^{\prime} c^{\prime \prime}\right\rangle\right\rangle
$$

For arbitrary 6-dimensional anisotropic quadratic forms in $I^{2}(k)$, the 2-Pfister number is 2, 3 or 4 . Note that scaling has an important effect on the Pfister number although it does not change the Stiefel-Whitney class. Indeed, by Theorem 2.2 and Remark 2.4, if $x_{1}, \ldots, x_{5}$ are independent indeterminates and

$$
q=\left\langle x_{1}, x_{2}, x_{3}, x_{4}, x_{5}, x_{1} x_{2} x_{3} x_{4} x_{5}\right\rangle,
$$

then

On the other hand,

$$
\operatorname{Pf}_{2}(q)=4 \quad \text { and } \quad \operatorname{Pf}_{2}\left(\left\langle x_{1}\right\rangle q\right)=3 .
$$

$$
\left\langle x_{1} x_{2} x_{3}\right\rangle q=\left\langle\left\langle x_{1} x_{2}, x_{1} x_{3}\right\rangle\right\rangle+\left\langle\left\langle x_{4} x_{5}, x_{1} x_{2} x_{3} x_{4}\right\rangle\right\rangle,
$$

hence

$$
\mathrm{Pf}_{2}\left(\left\langle x_{1} x_{2} x_{3}\right\rangle q\right)=2 .
$$

More generally, the same computation shows that for an arbitrary anisotropic form $q \in I^{2}(k)$ of dimension 6 , if $d \in k^{\times}$is the discriminant of some 3 -dimensional subform of $q$, then $\operatorname{Pf}_{2}(\langle d\rangle q)=2$. In the rest of this section, we give necessary and sufficient conditions on $q$ for $\operatorname{Pf}_{2}(q) \leq 3$ as well as for $\operatorname{Pf}_{2}(q)=2$.

As seen before, every quadratic form of dimension 6 in $I^{2}(k)$ is a scalar multiple of a form $q$ with $\operatorname{Pf}_{2}(q)=2$. Fix a decomposition

$$
q=\langle\langle a, b\rangle\rangle+\langle\langle c, d\rangle\rangle=\langle a, b, a b, c, d, c d\rangle .
$$

To this decomposition is associated the biquaternion algebra $D=(a, b)_{k} \otimes(c, d)_{k}$, which is Brauer-equivalent to the Clifford algebra of $q$, and the orthogonal involution $\sigma$ on $D$ that is the tensor product of the conjugation involutions on $(a, b)_{k}$ and $(c, d)_{k}$. The algebra $D$ is division since $q$ is anisotropic, see $[6,(16.5)]$. 
TheOrem 3.2. For $\lambda \in k^{\times}$, we have $\operatorname{Pf}_{2}(\langle\lambda\rangle q)=2$ if and only if $\lambda^{2}$ is the reduced norm of some $\sigma$-symmetric element in $D$, i.e.,

$$
\lambda^{2}=\operatorname{Nrd}_{D}(u) \quad \text { for some } u \in \operatorname{Sym}(D, \sigma) .
$$

PROOF. Let $(a, b)_{k}^{0}$ (resp. $\left.(c, d)_{k}^{0}\right)$ be the $k$-vector space of pure quaternions in $(a, b)_{k}$ (resp. in $\left.(c, d)_{k}\right)$. The vector space of $\sigma$-skew-symmetric elements in $D$ is

$$
\operatorname{Skew}(D, \sigma)=\left((a, b)_{k}^{0} \otimes 1\right) \oplus\left(1 \otimes(c, d)_{k}^{0}\right) .
$$

Let $p_{\sigma}$ be the linear operator on $\operatorname{Skew}(D, \sigma)$ defined by

$$
p_{\sigma}(x \otimes 1+1 \otimes y)=x \otimes 1-1 \otimes y \quad \text { for } x \in(a, b)_{k}^{0} \text { and } y \in(c, d)_{k}^{0} .
$$

The formula $q_{\sigma}(s)=s p_{\sigma}(s)$ defines a quadratic form on $\operatorname{Skew}(D, \sigma)$, and we have

$$
q_{\sigma} \simeq\langle a, b, a b, c, d, c d\rangle=q .
$$

Suppose now $\operatorname{Pf}_{2}(\langle\lambda\rangle q)=2$. We fix a decomposition

$$
\langle\lambda\rangle q=\left\langle\left\langle a^{\prime}, b^{\prime}\right\rangle\right\rangle+\left\langle\left\langle c^{\prime}, d^{\prime}\right\rangle\right\rangle=\left\langle a^{\prime}, b^{\prime}, a^{\prime} b^{\prime}, c^{\prime}, d^{\prime}, c^{\prime} d^{\prime}\right\rangle .
$$

The Clifford algebras of $q$ and $\langle\lambda\rangle q$ are isomorphic, hence we may identify

$$
D=\left(a^{\prime}, b^{\prime}\right)_{k} \otimes\left(c^{\prime}, d^{\prime}\right)_{k} .
$$

Let $\sigma^{\prime}$ be the orthogonal involution on $D$ that is the tensor product of the conjugation involutions on $\left(a^{\prime}, b^{\prime}\right)_{k}$ and $\left(c^{\prime}, d^{\prime}\right)_{k}$. By $[6,(2.7)]$, there is a unit $u \in \operatorname{Sym}(D, \sigma)$ such that $\sigma^{\prime}=\operatorname{Int}(u) \circ \sigma$, i.e., $\sigma^{\prime}(x)=u \sigma(x) u^{-1}$ for all $x \in D$. On $\operatorname{Skew}\left(D, \sigma^{\prime}\right)$ we may define a linear operator $p_{\sigma^{\prime}}$ and a quadratic form $q_{\sigma^{\prime}}$ in the same way as $p_{\sigma}$ and $q_{\sigma}$ were defined on $\operatorname{Skew}(D, \sigma)$, and we have

$$
q_{\sigma^{\prime}} \simeq\langle\lambda\rangle q .
$$

It is easily seen that $\operatorname{Skew}\left(D, \sigma^{\prime}\right)=u \operatorname{Skew}(D, \sigma)=\operatorname{Skew}(D, \sigma) u^{-1}$. The linear operator $p^{\prime}$ on $\operatorname{Skew}\left(D, \sigma^{\prime}\right)$ defined by

$$
p^{\prime}\left(s^{\prime}\right)=u p_{\sigma}\left(s^{\prime} u\right) \quad \text { for } s^{\prime} \in \operatorname{Skew}\left(D, \sigma^{\prime}\right)
$$

satisfies

$$
s^{\prime} p^{\prime}\left(s^{\prime}\right)=s^{\prime} u p_{\sigma}\left(s^{\prime} u\right)=q_{\sigma}\left(s^{\prime} u\right) \in k .
$$

Therefore, by $[6,(16.22)]$, the map $p^{\prime}$ is a multiple of $p_{\sigma^{\prime}}$ : there exists $\lambda_{1} \in k^{\times}$such that $p^{\prime}=\lambda_{1} p_{\sigma^{\prime}}$. It follows that

$$
s^{\prime} p^{\prime}\left(s^{\prime}\right)=\lambda_{1} q_{\sigma^{\prime}}\left(s^{\prime}\right) \quad \text { for } s^{\prime} \in \operatorname{Skew}\left(D, \sigma^{\prime}\right),
$$

and (7) shows that the map $s^{\prime} \mapsto s^{\prime} u$ is an isometry $\left\langle\lambda_{1}\right\rangle q_{\sigma^{\prime}} \simeq q_{\sigma}$. Hence $\left\langle\lambda_{1} \lambda\right\rangle q \simeq q$ and $\lambda \lambda_{1}^{-1}$ is the multiplier of a similitude of $q$. By $[\mathbf{6},(15.34)]$, we may find $\lambda_{2} \in k^{\times}$ and $v \in D^{\times}$such that

$$
\lambda \lambda_{1}^{-1}=\lambda_{2}^{2} \operatorname{Nrd}_{D}(v) .
$$

On the other hand, for $s^{\prime} \in \operatorname{Skew}\left(D, \sigma^{\prime}\right)$ we have $q_{\sigma}\left(s^{\prime} u\right)^{2}=\operatorname{Nrd}_{D}\left(s^{\prime} u\right)$ and $q_{\sigma^{\prime}}\left(s^{\prime}\right)^{2}=\operatorname{Nrd}_{D}\left(s^{\prime}\right)$ by $[6,(16.25)]$, hence $(7)$ and (8) yield

$$
\lambda_{1}^{2}=\operatorname{Nrd}_{D}(u)
$$

Using this equation, we derive from (9):

$$
\lambda^{2}=\lambda_{1}^{2} \lambda_{2}^{4} \operatorname{Nrd}_{D}(v)^{2}=\operatorname{Nrd}_{D}\left(\lambda_{2} v u \sigma(v)\right) .
$$

Since $\lambda_{2} v u \sigma(v) \in \operatorname{Sym}(D, \sigma)$, the element $\lambda$ satisfies the condition in the theorem. 
Conversely, assume $\lambda^{2}=\operatorname{Nrd}_{D}(u)$ for some $u \in \operatorname{Sym}(D, \sigma)$. Define an orthogonal involution $\sigma^{\prime}$ on $D$ by $\sigma^{\prime}=\operatorname{Int}(u) \circ \sigma$. By $[6,(7.3)]$, the discriminant of $\sigma^{\prime}$ is $\operatorname{Nrd}_{D}(u)=\lambda^{2}$, hence by $[6,(15.12)]$ we may find quaternion subalgebras $\left(a^{\prime}, b^{\prime}\right)_{k}$, $\left(c^{\prime}, d^{\prime}\right)_{k} \subseteq D$ such that

$$
D=\left(a^{\prime}, b^{\prime}\right)_{k} \otimes\left(c^{\prime}, d^{\prime}\right)_{k},
$$

and $\sigma^{\prime}$ is the tensor product of the conjugations on $\left(a^{\prime}, b^{\prime}\right)_{k}$ and $\left(c^{\prime}, d^{\prime}\right)_{k}$. We may then define $p_{\sigma^{\prime}}$ and $q_{\sigma^{\prime}}$ as above, and we have

$$
q_{\sigma^{\prime}} \simeq\left\langle a^{\prime}, b^{\prime}, a^{\prime} b^{\prime}, c^{\prime}, d^{\prime}, c^{\prime} d^{\prime}\right\rangle
$$

On the other hand, define a linear operator $p_{0}$ and a quadratic form $q_{0}$ on $\operatorname{Skew}\left(D, \sigma^{\prime}\right)$ by

$$
p_{0}\left(s^{\prime}\right)=\lambda^{-1} u p_{\sigma}\left(s^{\prime} u\right), \quad q_{0}\left(s^{\prime}\right)=\lambda^{-1} q_{\sigma}\left(s^{\prime} u\right) \quad \text { for } s^{\prime} \in \operatorname{Skew}\left(D, \sigma^{\prime}\right) .
$$

By definition, we have

$$
q_{0} \simeq\langle\lambda\rangle q_{\sigma} \simeq\langle\lambda\rangle q
$$

Moreover, $s^{\prime} p_{0}\left(s^{\prime}\right)=q_{0}\left(s^{\prime}\right) \in k$ for $s^{\prime} \in \operatorname{Skew}\left(D, \sigma^{\prime}\right)$, hence $p_{0}$ is a multiple of $p_{\sigma^{\prime}}$ by $[\mathbf{6},(16.22)]$ : we have $p_{0}=\mu p_{\sigma^{\prime}}$ for some $\mu \in k^{\times}$, hence also $q_{0}=\mu q_{\sigma^{\prime}}$. For $s^{\prime} \in \operatorname{Skew}\left(D, \sigma^{\prime}\right)$ we have by $[\mathbf{6},(16.25)]$

$$
p_{0}^{2}\left(s^{\prime}\right)=\lambda^{-2} u p_{\sigma}\left(u p_{\sigma}\left(s^{\prime} u\right) u\right)=\lambda^{-2} \operatorname{Nrd}_{D}(u) p_{\sigma}^{2}\left(s^{\prime} u\right) u^{-1} .
$$

Since $p_{\sigma}^{2}=\mathrm{Id}$ and $\operatorname{Nrd}_{D}(u)=\lambda^{2}$, it follows that $p_{0}^{2}=\mathrm{Id}$. Now, we also have $p_{\sigma^{\prime}}^{2}=\mathrm{Id}$, hence $\mu= \pm 1$. Therefore, $q_{0} \simeq\langle \pm 1\rangle q_{\sigma^{\prime}} \simeq q_{\sigma^{\prime}}$. By (10) and (11) we have

$$
\langle\lambda\rangle q \simeq\left\langle a^{\prime}, b^{\prime}, a^{\prime} b^{\prime}, c^{\prime}, d^{\prime}, c^{\prime} d^{\prime}\right\rangle,
$$

hence $\operatorname{Pf}_{2}(\langle\lambda\rangle q)=2$.

Note that the group $S(q) \subset k^{\times}$of spinor norms of $q$ can be described in terms of $D$ : we have by $[\mathbf{6},(15.34)]$

$$
S(q)=\left\{\lambda \in k^{\times} \mid \lambda^{2} \in \operatorname{Nrd}_{D}\left(D^{\times}\right)\right\} .
$$

Therefore, the following is a direct consequence of Theorem 3.2:

Corollary 3.3. Let $q \in I^{2}(k)$ be an anisotropic quadratic form of dimension 6, and let $\lambda \in k^{\times}$. If $\operatorname{Pf}_{2}(q)=\operatorname{Pf}_{2}(\langle\lambda\rangle q)=2$, then $\lambda$ is a spinor norm of $q$.

We now turn to a characterization of quadratic forms of dimension 6 with 2-Pfister number at most 3.

THEOREM 3.4. Let $q \in I^{2}(k)$ be an anisotropic quadratic form of dimension 6 . We have $\mathrm{Pf}_{2}(q) \leq 3$ if and only if there exist a 4-dimensional quadratic form $q_{1}$ over $k$ and scalars $\mu, \mu^{\prime}, \nu \in k^{\times}$satisfying the following conditions:

(i) $q \simeq q_{1} \perp\left\langle\mu^{\prime}\right\rangle\langle\langle\nu\rangle\rangle$;

(ii) $\operatorname{Pf}_{2}\left(q_{1} \perp\langle\mu\rangle\langle\langle\nu\rangle\rangle\right) \leq 2$;

(iii) $\left\langle\left\langle\mu, \mu^{\prime}, \nu\right\rangle\right\rangle=0$.

Proof. Suppose $\operatorname{Pf}_{2}(q) \leq 3$, and let $q=\left\langle\left\langle x_{1}, y_{1}\right\rangle\right\rangle+\left\langle\left\langle x_{2}, y_{2}\right\rangle\right\rangle+\left\langle\left\langle x_{3}, y_{3}\right\rangle\right\rangle=\left\langle x_{1}, y_{1}, x_{1} y_{1}, x_{2}, y_{2}, x_{2} y_{2}\right\rangle+\left\langle 1, x_{3}, y_{3}, x_{3} y_{3}\right\rangle$.

Since the dimension of $q$ is 6 , there exists a 2 -dimensional form $\langle\mu\rangle\langle\langle\nu\rangle$ that is a subform of $\left\langle x_{1}, y_{1}, x_{1} y_{1}, x_{2}, y_{2}, x_{2} y_{2}\right\rangle$ and of $\left\langle 1, x_{3}, y_{3}, x_{3} y_{3}\right\rangle$. Thus, we can write

$$
\left\langle x_{1}, y_{1}, x_{1} y_{1}, x_{2}, y_{2}, x_{2} y_{2}\right\rangle=q_{1} \perp\langle\mu\rangle\langle\langle\nu\rangle\rangle
$$


and

$$
\left\langle 1, x_{3}, y_{3}, x_{3} y_{3}\right\rangle=\left\langle\mu_{1}, \mu_{2}\right\rangle \perp\langle\mu\rangle\langle\langle\nu\rangle\rangle
$$

for some 4-dimensional quadratic form $q_{1}$ and some scalars $\mu_{1}, \mu_{2}$. Equation (12) readily yields (ii). Comparing discriminants on each side of (13), we see that

$$
\left\langle\mu_{1}, \mu_{2}\right\rangle=\left\langle\mu^{\prime}\right\rangle\langle\langle\nu\rangle\rangle \quad \text { for some } \mu^{\prime} \in k^{\times} \text {. }
$$

Therefore, adding (12) and (13) yields (i). Finally, (13) shows that $\left\langle\mu, \mu^{\prime}\right\rangle\langle\langle\nu\rangle\rangle$ represents 1 , hence $\left\langle 1, \mu, \mu^{\prime}, \mu \nu, \mu^{\prime} \nu\right\rangle$ is isotropic. Since this form is contained in the 3-fold Pfister form $\left\langle\left\langle\mu, \mu^{\prime}, \nu\right\rangle\right\rangle$, we have (iii).

Conversely, suppose (i), (ii), and (iii) hold for some 4-dimensional quadratic form $q_{1}$ and some scalars $\mu, \mu^{\prime}, \nu \in k^{\times}$. Since

$$
\left\langle\left\langle\mu, \mu^{\prime}, \nu\right\rangle\right\rangle=\left\langle\mu, \mu \nu, \mu^{\prime}, \mu^{\prime} \nu\right\rangle \perp\left\langle 1, \nu, \mu \mu^{\prime}, \mu \mu^{\prime} \nu\right\rangle,
$$

condition (iii) yields

$$
\langle\mu\rangle\langle\langle\nu\rangle\rangle\left\langle\left\langle\mu^{\prime}\right\rangle\langle\langle\nu\rangle\rangle=\left\langle\left\langle\nu, \mu \mu^{\prime}\right\rangle\right\rangle\right.
$$

Therefore, we derive from (i) that

$$
q=\left(q_{1} \perp\langle\mu\rangle\langle\langle\nu\rangle\rangle\right)+\left\langle\left\langle\nu, \mu \mu^{\prime}\right\rangle\right\rangle .
$$

Since $\operatorname{Pf}_{2}\left(q_{1} \perp \mu\langle\langle\nu\rangle\rangle\right) \leq 2$ by (ii), it follows that $\operatorname{Pf}_{2}(q) \leq 3$.

\section{References}

[1] J. Kr. Arason, Cohomologische Invarianten quadratischer Formen, J. Algebra 36 (1975), no. 3, 448-491.

[2] J. Kr. Arason, R. Elman and B. Jacob, Fields of cohomological 2-dimension three, Math. Ann. 274 (1986), no. 4, 649-657.

[3] P. Brosnan, Z. Reichstein and A. Vistoli, Essential dimension, spinor groups, and quadratic forms, to appear in Ann. Math.

[4] S. Garibaldi, A. Merkurjev and J.-P. Serre, Cohomological invariants in Galois cohomology, Amer. Math. Soc., Providence, RI, 2003.

[5] B. Kahn, On "horizontal" invariants attached to quadratic forms, pp. 21-33, Hindustan Book Agency, Delhi, 2005

[6] M.-A. Knus et al., The book of involutions, Amer. Math. Soc., Providence, RI, 1998.

[7] T. Y. Lam, Introduction to quadratic forms over fields, Amer. Math. Soc., Providence, RI, 2005.

[8] J. Milnor, Algebraic K-theory and quadratic forms, Invent. Math. 9 (1969/1970), 318-344.

[9] R. Parimala and V. Suresh, On the length of a quadratic form, in Algebra and number theory, pp. 147-157, Hindustan Book Agency, Delhi, 2005.

Department of Mathematics and Computer Science, Emory University, 400 Dowman Drive, Atlanta, Georgia 30322 USA

E-mail address: parimala@mathcs.emory.edu

Department of Mathematics and Statistics, University of Hyderabad, Gachibowli, HYDERABAD - 500046 INDIA

E-mail address: vssm@uohyd.ernet.in

Département de mathématique, Université Catholique de Louvain, Chemin du CyClotron, 2, B-1348 Louvain-La-Neuve, Belgium

E-mail address: jean-pierre.tignol@uclouvain.be 